\title{
Aperiodic Complementary Sets of Sequences-Based MIMO Frequency Selective Channel Estimation
}

\author{
Shuangquan Wang, Student Member, IEEE, and Ali Abdi, Member, IEEE
}

\begin{abstract}
Accurate estimation of MIMO frequency selective fading channels is important for reliable communication. In this letter, a new channel estimator which relies on aperiodic complementary sets of sequences is proposed. Theoretical analysis and Monte-Carlo simulation have shown that it achieves the minimum possible Cramér-Rao lower bound. A low-complexity hardware implementation of the estimator is also provided.
\end{abstract}

Index Terms-MIMO Channels, Aperiodic Complementary Sets of Sequences, Channel Estimation, Frequency Selective Fading.

\section{INTRODUCTION}

C HANNEL state information is required for coherent detection at the receiver, which gives rise to the need for accurate channel estimation. Training-based channel estimation is widely used in most existing communication systems because it offers less complexity and better performance, compared to the blind approach. Among various training sequences in literatures, the complementary pair [1] has a special place in single-input single-output channel estimation [2][3]. In this letter, we propose to use aperiodic complementary sets of sequences [4] for multiple-input multipleoutput (MIMO) frequency selective channel estimation, due to their low complexity and capability to achieve the minimum possible Cramér-Rao lower bound (CRLB).

Notation: ${ }^{T}$ is the matrix transpose, ${ }^{-1}$ is the matrix inverse, $\|\cdot\|_{F}$ represents the Frobenius norm, $\mathbb{E}[\cdot]$ is the mathematical expectation, $\cdot-$ is the sample average, $\mathbf{I}_{m}$ denotes the $m \times m$ identity matrix, $\mathbf{0}_{m \times n}$ is the $m \times n$ zero matrix, $\propto$ means proportional to, $\widehat{x}$ is the estimator of $x, t \in[m, n]$ implies $t$ is an integer such that $m \leqslant t \leqslant n,[\cdot]_{i, j}, i, j \geqslant 0$ is the $\{i, j\}^{\text {th }}$ entry of a matrix, $\operatorname{tr}[\cdot]$ is the trace of a matrix, $N_{T}$ and $N_{R}$ are the numbers of transmit and receive antennas, respectively, and $N_{x}$ is the length of each training sequence.

\section{Definition AND CONSTRUCTION OF APERIODIC COMPLEMENTARY SETS OF SEQUENCES}

Let $\mathbf{a}_{i}=\left[a_{i, 0}, a_{i, 1}, \cdots, a_{i,(N-1)}\right]$ be a sequence of \pm 1 's, and $r_{\mathbf{a}_{i}, \mathbf{a}_{i}}(k)=\sum_{n=0}^{N-1-|k|} a_{i, n} a_{i,(n+|k|)},|k| \in[0, N-1]$, is the aperiodic autocorrelation of the sequence $\mathbf{a}_{i}$ [4]. A set of $p$ sequences $\left\{\mathbf{a}_{i}\right\}_{i=0}^{p-1}$ is complementary if and only

Manuscript receivedApr. 20, 2005. The associated editor coordinating the review of this letter and approving it for publication was Dr. Keith Zhang.

The authors are with the Center for Wireless Communications and Signal Processing Research (CWCSPR), Department of Electrical and Computer Engineering, New Jersey Institute of Technology, Newark, NJ 07102 USA (e-mail: $\{$ sw27, ali.abdi $\} @$ njit.edu). if $\sum_{i=0}^{p-1} r_{\mathbf{a}_{i}, \mathbf{a}_{i}}(k)=0, k \neq 0$. In this letter, only sequences with the equal length $N$ are considered, therefore, $\sum_{i=0}^{p-1} r_{\mathbf{a}_{i}, \mathbf{a}_{i}}(0)=p N$. If another set of $p$ sequences $\left\{\mathbf{b}_{i}\right\}_{i=0}^{p-1}$ is complementary and $\sum_{i=0}^{p-1} r_{\mathbf{a}_{i}, \mathbf{b}_{i}}(k)=0, \forall k$, where $r_{\mathbf{a}_{i}, \mathbf{b}_{i}}(k)=\sum_{n=0}^{N-1-k} a_{i, n} b_{i,(n+k)}, k \in[0, N-1]$ and $r_{\mathbf{a}_{i}, \mathbf{b}_{i}}(k)=r_{\mathbf{b}_{i}, \mathbf{a}_{i}}(-k), k \in[-N+1,-1]$, then $\left\{\mathbf{b}_{i}\right\}_{i=0}^{p-1}$ is a mate of $\left\{\mathbf{a}_{i}\right\}_{i=0}^{p-1}$. More details can be found in [4].

A collection of aperiodic complementary sets of sequences $\left\{\mathbf{a}_{i}\right\}_{i=0}^{p-1},\left\{\mathbf{b}_{i}\right\}_{i=0}^{p-1}, \cdots,\left\{\mathbf{z}_{i}\right\}_{i=0}^{p-1}$ are mutually uncorrelated if every two aperiodic complementary sets of sequences in the collection are mates of each other.

In this letter, we take $p=2$. The aperiodic complementary sequence pair $\left\{\mathbf{a}_{0}, \mathbf{a}_{1}\right\}$ with $N=2^{M}, M \geqslant 1$, can be constructed by the following recursive method [5]

$$
\begin{aligned}
& a_{0, k}^{(m)}=a_{0, k}^{(m-1)}+a_{1,\left(k-2^{M-m}\right)}^{(m-1)}, \quad m \in[1, M], \\
& a_{1, k}^{(m)}=a_{0, k}^{(m-1)}-a_{1,\left(k-2^{M-m}\right)}^{(m-1)}
\end{aligned}
$$

with $a_{0, k}^{(0)}=a_{1, k}^{(0)}=\delta_{k}$, where $\delta_{0}=1, \delta_{k}=0, k \neq 0$. After $M$ iterations, we obtain a pair of aperiodic complementary sequences $\mathbf{a}_{0}=\left[a_{0,0}, a_{0,1}, \cdots, a_{0, N-1}\right]$ and $\mathbf{a}_{1}=$ $\left[a_{1,0}, a_{1,1}, \cdots, a_{1, N-1}\right]$, each of length $N$.

Based on property 3 ) in [1], $\mathbf{a}_{0}$ and $\overleftarrow{\mathbf{a}_{1}}$ are also complementary, where $\overleftarrow{\mathbf{b}}$ is the reverse of the sequence $\mathbf{b}$, i.e., $\overleftarrow{b}_{k}=b_{N-1-k}, k \in[0, N-1]$. Moreover, according to Theorem 11 in [4], if $\{\mathbf{a}, \mathbf{b}\}$ is a complementary set of two sequences of length $N$, then $\{\overleftarrow{\mathbf{b}},-\overleftarrow{\mathbf{a}}\}$ is its mate. So, $\left\{\mathbf{a}_{1},-\overleftarrow{\mathbf{a}_{0}}\right\}$ is the mate of $\left\{\mathbf{a}_{0}, \overleftarrow{\mathbf{a}_{1}}\right\}$, and vice versa.

\section{SySTEM AND CHANNEL MODELS}

We consider the block fading model for frequency selective MIMO channels, where the channel response is fixed within one block and changes from one block to another, randomly. This is a suitable model for indoor MIMO channels due to the low mobility [6]. In what follows, we consider an $L+1$ tap channel impulse response (CIR) with the tap index $l \in[0, L]$, the transmit antenna index $n_{t} \in\left[1, N_{T}\right]$, the receive antenna index $n_{r} \in\left[1, N_{R}\right]$, the training symbol index $n \in\left[0, N_{x}-1\right]$, and the signal index at the receiver $k \in\left[0, N_{x}+L-1\right]$.

Let $\mathbf{H}=\left[\begin{array}{llll}\mathbf{H}_{0} & \mathbf{H}_{1} & \cdots & \mathbf{H}_{L}\end{array}\right]$ be the CIR matrix of the MIMO frequency selective channel, where

$$
\mathbf{H}_{l}=\left[\begin{array}{ccc}
h_{1,1}(l) & \cdots & h_{1, N_{T}}(l) \\
\vdots & \ddots & \vdots \\
h_{N_{R}, 1}(l) & \cdots & h_{N_{R}, N_{T}}(l)
\end{array}\right]
$$

in which $h_{n_{r}, n_{t}}(l)$ is the $l^{\text {th }}$ tap of the CIR between the $n_{r}^{\text {th }}$ receive antenna and the $n_{t}^{\text {th }}$ transmit antenna. Using matrix 
notation, the signals received by $N_{R}$ antennas, corresponding to the training symbols transmitted from $N_{T}$ antennas, can be written as

$$
\mathbf{Y}=\sqrt{\rho / N_{T}} \mathbf{H X}+\mathbf{E},
$$

where the training matrix $\mathbf{X}$, whose dimension is $N_{T}(L+1) \times$ $\left(N_{x}+L\right)$, is defined by

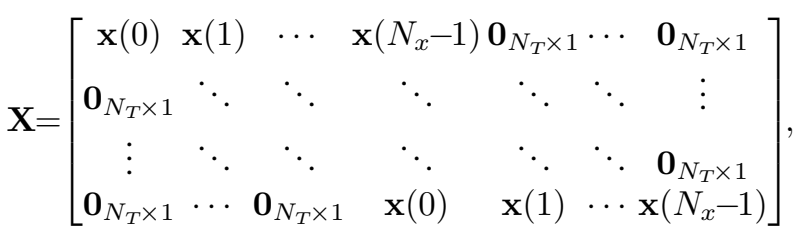

in which $\mathbf{x}(n)=\left[x_{1}(n), x_{2}(n), \cdots, x_{N_{T}}(n)\right]^{T}$. Note that $\mathbf{Y}=\left[\mathbf{y}(0), \mathbf{y}(1), \cdots, \mathbf{y}\left(N_{x}+L-1\right)\right]$, where $\mathbf{y}(k)=$ $\left[y_{1}(k), y_{2}(k), \cdots, y_{N_{R}}(k)\right]^{T}$. The noise matrix is defined as $\mathbf{E}=\left[\mathbf{e}(0), \mathbf{e}(1), \cdots, \mathbf{e}\left(N_{x}+L-1\right)\right]$, where $\mathbf{e}(k)=$ $\left[e_{1}(k), e_{2}(k), \cdots, e_{N_{R}}(k)\right]^{T}$.

Clearly $x_{n_{t}}(n)$ is the training symbol transmitted from the $n_{t}^{\text {th }}$ transmit antenna at time $n, y_{n_{r}}(k)$ is the signal received by the $n_{r}^{\text {th }}$ receive antenna at time $k$, polluted by the zero-mean and unit variance additive complex Gaussian noise component $e_{n_{r}}(k)$, and $\rho$ is the expected received signal-to-noise ratio (SNR) at each receive antenna. Each subchannel is normalized to have unit power, i.e., $\sum_{l=0}^{L} \mathbb{E}\left[\left|h_{n_{r}, n_{t}}(l)\right|^{2}\right]=1, \forall n_{r}, n_{t}$.

\section{Construction of Optimal Training Sequences}

When the elements of $\mathbf{E}$ in (3) are uncorrelated (spatiotemporal white noise) and Gaussian, and $\mathbf{H}$ is considered as an unknown deterministic matrix, then the maximum likelihood estimator (MLE) of $\mathbf{H}$ is the same as the least square estimator (LSE), given by [7]

$$
\widehat{\mathbf{H}}=\sqrt{N_{T} / \rho} \mathbf{Y} \mathbf{X}^{T}\left(\mathbf{X X}^{T}\right)^{-1} .
$$

This estimator achieves the CRLB, therefore is efficient, and the total mean square error (MSE) is $\mathbb{E}\left[\|\widehat{\mathbf{H}}-\mathbf{H}\|_{F}^{2}\right]=$ $\frac{N_{T} N_{R}}{\rho} \operatorname{tr}\left[\left(\mathbf{X X}^{T}\right)^{-1}\right]$. In order to achieve the minimum possible total MSE under the training power constraint, the training sequences should satisfy the condition $\mathbf{X X}^{T} \propto \mathbf{I}$ [7][8]. To satisfy this condition, the training sequence from each antenna has to be orthogonal not only to its temporal shifts within $L$ taps, but also to the training sequences from other antennas and their shifts within $L$ taps. Since $\mathbf{X}$ has a blockToeplitz structure, it is hard to find such training sequences $[7]^{1}$. However, we can construct two training matrices such that $\mathbf{X}_{1} \mathbf{X}_{1}^{T}+\mathbf{X}_{2} \mathbf{X}_{2}^{T} \propto \mathbf{I}$, by using aperiodic complementary sets of sequences of Sec. II, where the indices $[\cdot]_{1}$ and $[\cdot]_{2}$ correspond to the preamble and postamble parts of the twosided training configuration, respectively, as shown in Fig. 1. The frame structure is the same for all Tx antennas. The "Gap"

\footnotetext{
${ }^{1}$ The ZCZ sequences [9], mentioned by one reviewer, are developed based on periodic correlations, which are not the focus of this letter.
}

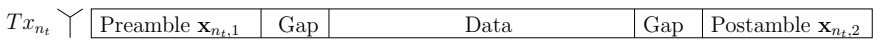

Fig. 1. Frame structure for $n_{t}^{\text {th }}$ transmit antenna in a MIMO system.
TABLE I

AssignMEnt of Training Symbols to TRANSmit Antennas

\begin{tabular}{|c||c|c|}
\hline$T_{x}$ & $\mathbf{x}_{n_{t}, 1}$ & $\mathbf{x}_{n_{t}, 2}$ \\
\hline \hline 1 & $\mathbf{u}$ & $\mathbf{v}^{\prime}$ \\
\hline 2 & $\mathbf{v}$ & $-\mathbf{u}^{\prime}$ \\
\hline 3 & $\mathbf{u} \Pi^{L+1}$ & $\mathbf{v}^{\prime} \Pi^{L+1}$ \\
\hline 4 & $\mathbf{v} \Pi^{L+1}$ & $-\mathbf{u}^{\prime} \Pi^{L+1}$ \\
\hline$\vdots$ & $\vdots$ & $\vdots$ \\
\hline$N_{T}-1$ & $\mathbf{u} \Pi^{q}$ & $\mathbf{v}^{\prime} \Pi^{q}$ \\
\hline$N_{T}$ & $\mathbf{v} \Pi^{q}$ & $-\mathbf{u}^{\prime} \Pi^{q}$ \\
\hline
\end{tabular}

of $L 0$ 's is required to separate the training and data symbols. In addition, $L 0$ 's are needed between two consecutive frames to avoid the inter-frame interference.

If $N_{T}$ is odd, we can add one virtual antenna to make it even. So, in what follows, we assume $N_{T}$ is even, without loss of generality. Let $\left\{\mathbf{a}_{0}, \mathbf{a}_{1}\right\}$ be a pair of complementary sequences generated by (1). Note that the complementary property will not change if we append some 0's to the beginning or end or both sides of the complementary pair. We define four row vector of length $N_{x}$ as $\mathbf{u}=\left[\mathbf{a}_{0}, \mathbf{o}\right], \mathbf{v}=$ $\left[\mathbf{a}_{1}, \mathbf{o}\right], \mathbf{u}^{\prime}=\left[\overleftarrow{\mathbf{a}_{0}}, \mathbf{o}\right]$, and $\mathbf{v}^{\prime}=\left[\overleftarrow{\mathbf{a}_{1}}, \mathbf{o}\right]$, where $\mathbf{o}=\mathbf{0}_{1 \times q}$ and $q=\left(\frac{N_{T}}{2}-1\right)(L+1)$. Note that $\mathbf{a}_{0}$ and $\mathbf{a}_{1}$ are generated by (1), therefore, $N_{x}=N+q$. Based on the properties discussed in Sec. II, $\left\{\mathbf{u}, \mathbf{v}^{\prime}\right\}$ and $\left\{\mathbf{v},-\mathbf{u}^{\prime}\right\}$ are mutually uncorrelated.

The training symbol assignment is given in Table I, where $\mathbf{x}_{n_{t}, 1}$ and $\mathbf{x}_{n_{t}, 2}$ are shown in Fig. 1, $\boldsymbol{\Pi}$ is the forward shift permutation matrix of order $N_{x}$ [10, p. 27], and $\mathbf{x} \Pi^{p}$ shifts the sequence $\mathbf{x}$ cyclically to the right by $p$ elements.

When using both preamble and postamble symbols, (3) can be written as

$$
\left[\begin{array}{ll}
\mathbf{Y}_{1} & \mathbf{Y}_{2}
\end{array}\right]=\sqrt{\gamma / N_{T}} \mathbf{H}\left[\begin{array}{ll}
\mathbf{X}_{1} & \mathbf{X}_{2}
\end{array}\right]+\left[\begin{array}{ll}
\mathbf{E}_{1} & \mathbf{E}_{2}
\end{array}\right],
$$

where $\gamma$ is set to $\frac{N_{x}}{N} \rho$, as a compensation for inserting $q 0$ 's into the original \pm 1 complementary sequences, to keep the SNR at each receive antenna equal to $\rho$. Based on (5) and the independence of $\mathbf{E}_{1}$ and $\mathbf{E}_{2}$, the LSE of $\mathbf{H}$ is given by

$$
\widehat{\mathbf{H}}=\sqrt{\frac{N_{T}}{\gamma}}\left(\sum_{t=1}^{2} \mathbf{Y}_{t} \mathbf{X}_{t}^{T}\right)\left(\sum_{t=1}^{2} \mathbf{X}_{t} \mathbf{X}_{t}^{T}\right)^{-1} .
$$

According to (4) and Table I, it is easy to show that $\mathbf{X}_{1} \mathbf{X}_{1}^{T}+$ $\mathbf{X}_{2} \mathbf{X}_{2}^{T}=2 N \mathbf{I}_{N_{T}(L+1)}$. This demonstrates the optimality of our design and simplifies (7) to

$$
\widehat{\mathbf{H}}=\frac{\sqrt{N_{T}}}{2 N \sqrt{\gamma}} \sum_{t=1}^{2} \mathbf{Y}_{t} \mathbf{X}_{t}^{T} .
$$

\section{FAST IMPLEMENTATION OF THE CHANNEL ESTIMATOR}

Let us define a $2 \times \frac{N_{T}(L+1)}{2}$ matrix ${ }_{n_{r}} \mathbf{H}$ that includes the CIR of all the subchannels between $N_{T}$ transmit antennas and the $n_{r}^{\text {th }}$ receive antenna, as follows

$$
{ }_{n_{r}} \mathbf{H}=\left[\begin{array}{llll}
{ }_{n_{r}}^{1} \mathbf{H} & { }_{n_{r}}^{2} \mathbf{H} & \cdots & \frac{N_{T}}{n_{r}^{2}} \mathbf{H}
\end{array}\right],
$$




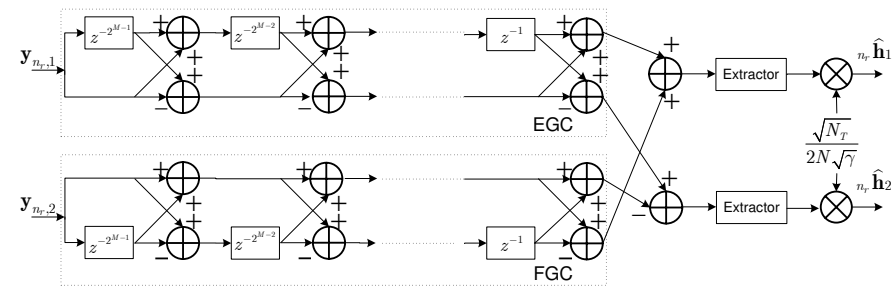

Fig. 2. The fast estimator on the $n_{r}^{\text {th }}$ receive antenna.

where ${ }_{n_{r}}^{s} \mathbf{H}=\left[\begin{array}{cccc}h_{n_{r}, 2 s-1}(0) & h_{n_{r}, 2 s-1}(1) & \cdots & h_{n_{r}, 2 s-1}(L) \\ h_{n_{r}, 2 s}(0) & h_{n_{r}, 2 s}(1) & \cdots & h_{n_{r}, 2 s}(L)\end{array}\right], s \in\left[1, \frac{N_{T}}{2}\right]$. Based on (8) and (9), we can write the estimator for ${ }_{n_{r}} \mathbf{H}$ as

$$
{ }_{n_{r}} \widehat{\mathbf{H}}=\frac{\sqrt{N_{T}}}{2 N \sqrt{\gamma}} \sum_{t=1}^{2} \mathbf{S}_{t} \mathbf{Y}_{n_{r}, t},
$$

where the two $2 \times N$ matrices $\mathbf{S}_{1}$ and $\mathbf{S}_{2}$ are given by $\left[\begin{array}{l}\mathbf{a}_{0} \\ \mathbf{a}_{1}\end{array}\right]$ and $\left[\begin{array}{c}\overleftarrow{\mathbf{a}_{1}} \\ -\overleftarrow{\mathbf{a}_{0}}\end{array}\right]$, respectively, and $\mathbf{Y}_{n_{r}, t}, t \in[1,2]$ is the following Hankel matrix

$$
\mathbf{Y}_{n_{r}, t}=\left[\begin{array}{cccc}
y_{n_{r}, t}(0) & y_{n_{r}, t}(1) & \cdots & y_{n_{r}, t}\left(N_{x}+L-N\right) \\
y_{n_{r}, t}(1) & \ddots & \ddots & y_{n_{r}, t}\left(N_{x}+L-N+1\right) \\
\vdots & \ddots & \ddots & \vdots \\
y_{n_{r}, t}(N-1) & y_{n_{r}, t}(N) & \cdots & y_{n_{r}, t}\left(N_{x}+L-1\right)
\end{array}\right] \text {, }
$$

where $y_{n_{r}, 1}(k)$ and $y_{n_{r}, 2}(k)$ are the signals received by the $n_{r}^{\text {th }}$ receive antenna at time $k$, and correspond to the preamble and postamble, respectively. According to (10), clearly the structure of the estimator is identical for all the receive antennas, so we focus on the $n_{r}^{\text {th }}$ antenna in the sequel.

Based on the definitions of $\mathbf{S}_{1}$ and $\mathbf{Y}_{n_{r}, t}$, we see $\left[\mathbf{S}_{1} \mathbf{Y}_{n_{r}, 1}\right]_{i, j}=\sum_{n=0}^{N-1} a_{i, n} y_{n_{r}, 1}(n+j), j \in\left[0, \frac{N_{T}(L+1)}{2}-1\right]$, $i \in[0,1]$. By defining the new index $w=N-1-n$, we have $\left[\mathbf{S}_{1} \mathbf{Y}_{n_{r}, 1}\right]_{i, j}=\sum_{w=0}^{N-1} \overleftarrow{a_{i, w}} y_{n_{r}, 1}(N-1+j-w)$, where $\overleftarrow{a}_{i, w}=a_{i, N-1-w}, w \in[0, N-1]$. So the elements of $\mathbf{S}_{1} \mathbf{Y}_{n_{r}, 1}$ in (10) are the convolution of $\mathbf{y}_{n_{r}, 1}$ with $\overleftarrow{\mathbf{a}_{0}}$ and $\overleftarrow{\mathbf{a}_{1}}$, where $\mathbf{y}_{n_{r}, t}=\left[y_{n_{r}, t}(0), y_{n_{r}, t}(1), \cdots, y_{n_{r}, t}\left(N_{x}+L-1\right)\right], t \in[1,2]$. Therefore, we can feed the received vector $\mathbf{y}_{n_{r}, 1}$ into a finite impulse response (FIR) filter bank (consisting of two FIR filters), with impulse responses $\overleftarrow{\mathbf{a}_{0}}$ and $\overleftarrow{\mathbf{a}_{1}}$, to calculate the $1^{\text {st }}$ and $2^{\text {nd }}$ rows of $\mathbf{S}_{1} \mathbf{Y}_{n_{r}, 1}$, respectively. With the same reasoning, $\mathbf{S}_{2} \mathbf{Y}_{n_{r}, 2}$ can be computed by feeding $\mathbf{y}_{n_{r}, 2}$ into an FIR filter bank whose impulse responses are $\mathbf{a}_{1}$ and $-\mathbf{a}_{0}$.

Based on the above explanation, the elements of $\mathbf{S}_{1} \mathbf{Y}_{n_{r}, 1}$ and $\mathbf{S}_{2} \mathbf{Y}_{n_{r}, 2}$ can be obtained by four FIR filters. The conventional implementation of each filter needs $N-1$ complex adders. In what follows, we introduce a less complex hardware implementation, by utilizing the special structure of our proposed training sequences.

In order to convolve the inputs $\mathbf{y}_{n_{r}, 1}$ and $\mathbf{y}_{n_{r}, 2}$ with $\left\{\overleftarrow{\mathbf{a}_{0}}, \overleftarrow{\mathbf{a}_{1}}\right\}$ and $\left\{\mathbf{a}_{1},-\mathbf{a}_{0}\right\}$ to obtain the elements of $\mathbf{S}_{1} \mathbf{Y}_{n_{r}, 1}$ and $\mathbf{S}_{2} \mathbf{Y}_{n_{r}, 2}$ in (10), respectively, we propose to use the efficient Golay correlator (EGC) [11, Fig. 2] and the fast Golay correlator (FGC) [5, Fig. 1]. The efficient hardware scheme to implement (10) is shown in Fig. 2, where " $\bigoplus$ " is the complex adder, " $\bigotimes$ " is the multiplier with one complex input and one real input, " $z^{-D}$ " is the delay unit of length $D$, the "Extractor" discards the first and the last $N-1$ elements and keeps the middle part, and ${ }_{n_{r}} \widehat{\mathbf{h}}_{t}$ is the $t^{\text {th }}$ row of ${ }_{n_{r}} \widehat{\mathbf{H}}, t=1,2$. If one

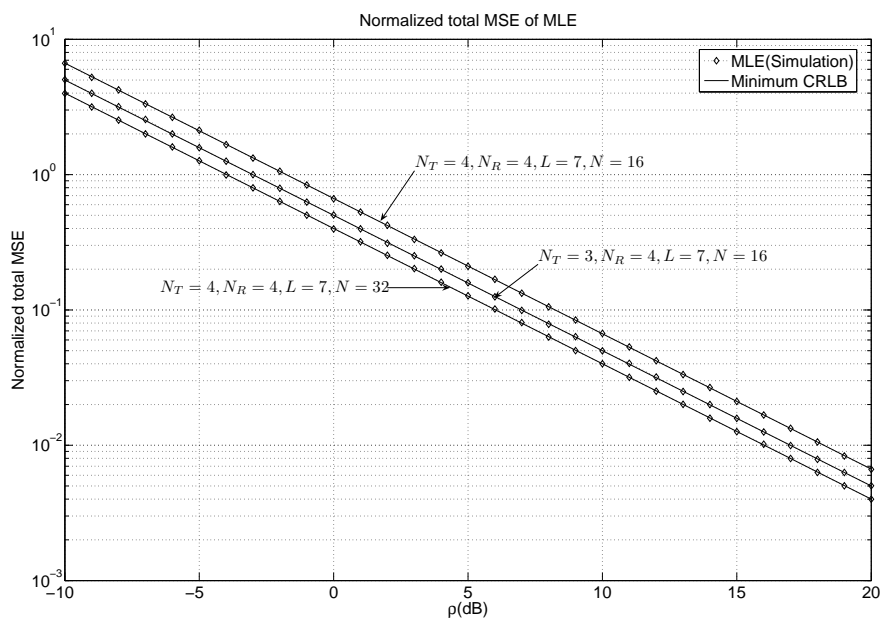

Fig. 3. The normalized total MSE of the proposed channel estimator and the minimum CRLB.

virtual antenna is added to make $N_{T}$ even, the last $L+1$ values in ${ }_{n} \widehat{\mathbf{h}}_{2}$ should be discarded, as they do not contain useful information.

Regarding the hardware complexity, according to Fig. 2, there are $4 M+2=4 \log _{2} N+2$ "Ð” units on each receive antenna. However, there are $4(N-1)+2$ “ $\bigoplus$ " units if (10) is implemented by the conventional method.

\section{Simulation Results AND Conclusion}

In Fig. 3 we have shown the minimum possible CRLB, normalized by $\mathbb{E}\left[\|\mathbf{H}\|_{F}^{2}\right]=N_{R} N_{T}$, which is given by $\frac{N_{T}(L+1)}{2 \gamma N}$, versus different SNR levels $\rho$, for three different scenarios. For each scenario, the simulated total MSE of the channel estimator in (8), $\overline{\|\mathbf{H}-\mathbf{H}\|_{F}^{2}}$, normalized by $\overline{\|\mathbf{H}\|_{F}^{2}}$, is also plotted in Fig. 3. As the simulation results demonstrate, the proposed estimator achieves the minimum CRLB. Moreover, the proposed fast hardware implementation makes it suitable for, but not limited to, practical MIMO-OFDM and MIMOUWB systems.

\section{REFERENCES}

[1] M. J. E. Golay, "Complementary series," IEEE Trans. Inform. Theory, vol. 7, pp. 82-87, Apr. 1961.

[2] P. Spasojevic and C. N. Georghiades, "Complementary sequences for ISI channel estimation," IEEE Trans. Inform. Theory, vol. 47, pp. 11451152, Mar. 2001

[3] B. Xu and G. Bi, "Channel estimation using complementary sequence pairs for UWB/OFDM systems," Electron. Lett., vol. 40, pp. 1196-1197, Sept. 2004.

[4] C. C. Tseng and C. L. Liu, "Complementary set of sequences," IEEE Trans. Inform. Theory, vol. 18, pp. 644-652, Sept. 1972.

[5] S. Z. Budišin, "Efficient pulse compressor for Golay complementary sequences," Electron. Lett., vol. 27, pp. 219-220, Jan. 1991.

[6] S. Wang, K. Raghukumar, A. Abdi, J. Wallace, and M. Jensen, "Indoor MIMO channels: A parametric correlation model and experimental results," in Proc. IEEE Sarnoff Symp., Princeton, NJ, 2004, pp. 1-5.

[7] E. G. Larsson and P. Stoica, Space-Time Block Coding for Wireless Communications. Cambridge, UK: Cambridge University Press, 2003.

[8] B. Hassibi and B. M. Hochwald, "How much training is needed in multiple-antenna wireless links?" IEEE Trans. Inform. Theory, vol. 49, pp. 951-963, Apr. 2003.

[9] P. Fan and W. H. Mow, "On optimal training sequence design for multiple-antenna systems over dispersive fading channels and its extensions," IEEE Trans. Veh. Technol., vol. 53, pp. 1623-1626, Sept. 2004.

[10] P. J. Davis, Circulant Matrices. New York: Wiley, 1979.

[11] B. M. Popović, "Efficient Golay correlator," Electron. Lett., vol. 35, pp. 1427-1428, Aug. 1999. 